\title{
MORFOLOGÍA E HISTOLOGÍA DE OSTEODERMOS DE UN PEIROSAURIDAE DE LA CUENCA NEUQUINA
}

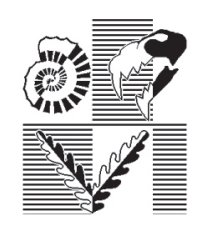

\section{LEONARDO SEBASTIÁN FILIPPI ${ }^{1}$, IGNACIO ALEJANDRO CERDA² Y ALBERTO CARLOS GARRIDO ${ }^{3}$}

${ }^{1}$ Museo Municipal “Argentino Urquiza”, Jujuy y Chaco s/n, Q8319BFA Rincón de los Sauces, Argentina. lsfilippi@yahoo.com.ar

${ }^{2}$ Museo de Geología y Paleontología, Universidad Nacional del Comahue, Buenos Aires 1400, Q8300IBX Neuquén, Argentina - CONICET-INIBIOMA. nachocerda6@yahoo.com.ar

${ }^{3}$ Museo Provincial de Ciencias Naturales "Prof. Dr. Juan Olsacher”. Dirección Provincial de Minería, Etcheluz y Ejército Argentino, Q8340AUD Zapala, Argentina. algene@copelnet.com.ar

Resumen. Se describen osteodermos de un nuevo ejemplar de crocodyliforme hallado en un afloramiento correspondiente a niveles de la Formación Plottier (Coniaciano tardío) de la localidad de Puesto Hernández, en inmediaciones de la localidad de Rincón de los Sauces, Provincia del Neuquén. Los osteodermos estudiados, que son parte del material hallado, están integrados por: región dorso-sacra, osteodermos apendiculares asociados al fémur derecho, región proximoventral de osteodermos caudales articulados y osteodermos aislados. La histología de los osteodermos permitió determinar que están constituidos por una corteza compacta que circunda una región interna más porosa. Fue posible reconocer marcas de crecimiento (annuli) en prácticamente todo el tejido compacto, pudiendo determinarse una edad mínima de 18 ańos para el espécimen estudiado. Los osteodermos exhiben caracteres que permiten preliminarmente asignar al ejemplar MAU-Pv-PH-437, como un Mesoeucrocodylia cercanamente relacionado con Peirosauridae.

Palabras clave. Crocodyliforme. Mesoeucrocodylia. Osteodermos. Histología. Cuenca Neuquina. Formación Plottier. Cretácico Superior.

Abstract. MORPHOLOGY AND HISTOLOGY OF OSTEODERMS FROM A PEIROSAURID FROM THE NEUQUEN BASIN. Osteoderms of a new crocodyliform specimen recovered from the Puesto Hernández locality are described here, found in sediments of the Plottier Formation (late Coniacian), near Rincón de los Sauces city, Neuquén Province. The studied osteoderms, that are part of the associated material, include: dorso-sacral region, appendicular osteoderms associated to the right femur, proximoventral region of articulated caudal osteoderms, and isolated osteoderms. The histology of the osteoderms allowed determining they have a compact cortex that surrounds a more cancellous internal region. It was possible to recognize growth marks (annuli) in the entire compact tissue, indicating a minimum age of 18 years for the studied specimen. The osteoderms have characters that allow preliminarily assigning the specimen MAU-Pv-PH-437 to Mesoeucrocodylia closely related to Peirosauridae.

Key words. Crocodyliformes. Mesoeucrocodylia. Osteoderms. Histology. Neuquén Basin. Plottier Formation. Upper Cretaceous.

Los crocodyliformes han sido estudiados anatómica y filogenéticamente casi exclusivamente sobre la base de materiales craneanos, por lo tanto, en la mayoría de los casos, los materiales postcraneanos de ejemplares fósiles no han sido descriptos exhaustivamente. No obstante, en los últimos años los caracteres postcraneanos han sido considerados en análisis cladísticos, manifestando así su valor taxonómico, filogenético y evolutivo (Clark, 1994; Pol, 1999, 2003, Pol y Norell, 2004; Ortega et al., 2000; Sereno et al., 2003, Carvalho et al., 2004, Turner, 2006; Fiorelli y Calvo, 2007, 2008; Pol et al., 2009; Sereno y Larsson, 2009). En relación con el análisis de materiales postcraneanos, los trabajos de descripción detallada de osteodermos en crocodyliformes (en algunos casos potenciados por la falta de buenos registros) son relativamente escasos (Wu et al., 1996; Salisbury y Frey, 2001; Salisbury et al., 2003; Marinho et al., 2006; Hill y Lucas, 2006; Pierce y Benton, 2006; Frey y Salisbury, 2007; Hill, 2010). Es por ello que el estudio mor- fológico e histológico de estos elementos, resulta significativo ya que aporta información relevante desde el punto de vista anatómico y permite además, hacer inferencias filogenéticas.

En el presente trabajo se describen osteodermos que corresponden a un crocodyliforme semiarticulado (MAU-Pv$\mathrm{PH}-437)$, cuyos materiales del esqueleto axial y apendicular serán objeto de análisis en un trabajo posterior. La preservación de osteodermos, íntimamente asociados a los restos postcraneanos del esqueleto axial y apendicular de este nuevo crocodyliforme del Cretácico Tardío del Norte de la Cuenca Neuquina, permitió realizar una descripción detallada de su morfología e histología, como así también, abordar interpretaciones respecto de la posición anatómica de cada uno de los morfotipos identificados. Al mismo tiempo, aporta significativa información que permite ampliar el conocimiento actual del esqueleto dérmico de los Crocodyliformes de la Cuenca Neuquina. 


\section{PALEONTOLOGÍA SISTEMÁTICA}

Superorden Crocodylomorpha Hay, 1930 (sensu Walker, 1970)

Orden Crocodyliformes Hay, 1930 (sensu Benton y Clark, 1988)

Suborden Mesoeucrocodylia

Whetstone y Whybrow, 1983

Familia Peirosauridae Gasparini, 1982

Peirosauridae indet.

Figuras 1-5

Material. MAU-Pv-PH-437 (Figs. 1-5), un diente completo y un fragmento de corona; ulna, radio y húmero izquierdos; escápula; tibia izquierda; tibia-fíbula, fémur asociado a osteodermos apendiculares, metatarsianos? derechos, astrágalo derecho?; cintura pélvica completa, secuencia articulada de vértebras dorsales posteriores, asociado a región dorso-sacra de osteodermos articulados, cuerpos vertebrales caudales articulados, región proximoventral de osteodermos caudales articulados, osteodermos aislados de diversa morfología y tamaño; costillas; arcos hemales y otros huesos indeterminados.

Procedencia geográfica. Yacimiento Hidrocarburífero Puesto Hernández, $30 \mathrm{~km}$ al oeste de la ciudad de Rincón de los Sauces, Provincia del Neuquén $\left(37^{\circ} 15^{\prime} 15^{\prime \prime} S-69^{\circ} 06^{\prime} 14^{\prime \prime} \mathrm{O}\right)$.

Procedencia estratigráfica. Formación Plottier (Grupo

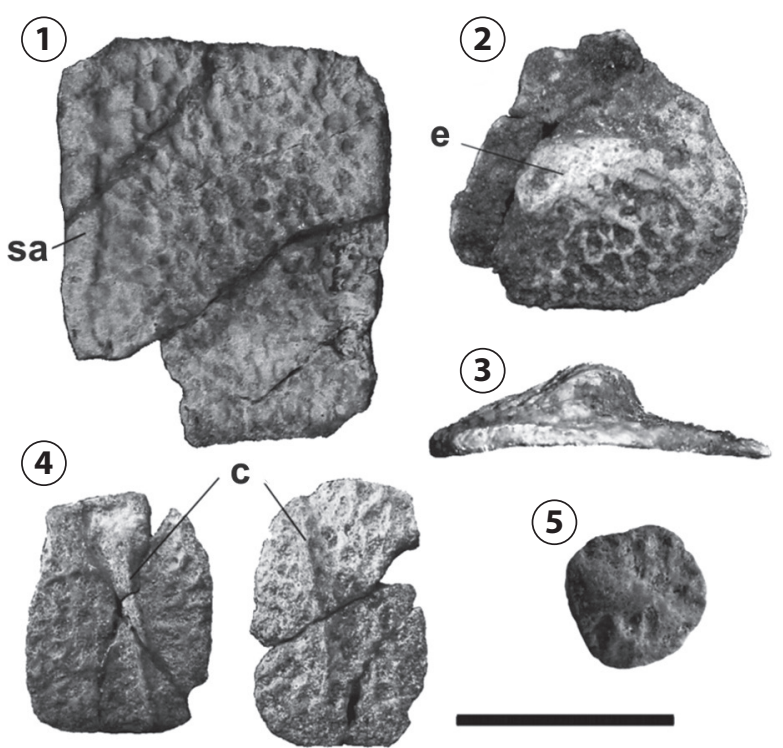

Figura 1. Osteodermos aislados.1, vista dorsal de osteodermo dorsal; 2, vista dorsal de osteodermos ?dorsal; 3, vista lateral de osteodermos? dorsal; 4, vista dorsal de osteodermos caudales accesorios; $\mathbf{5}$, vista dorsal de osteodermo apendicular. / Isolated osteoderm. 1, dorsal view of dorsal osteoderm; 2, dorsal view of ?dorsal osteoderm; 3, lateral view of ?dorsal osteoderm; $\mathbf{4}$, dorsal view of accesory caudal osteoderms; 5, dorsal view of apendicular osteoderm. Abreviaturas: c, cresta; e, espina; fa, faceta articular/ Abbreviations: c, keel; e, spine; fa, articular surface. Escala $/$ Scale $=2 \mathrm{~cm}$.
Neuquén, Subgrupo Río Colorado), Coniaciano tardío, Cretácico Tardío (Legarreta y Gulisano, 1989).

Descripción. Aunque la preservación del ejemplar no es óptima, los elementos dérmicos recuperados permiten realizar una detallada descripción de su morfología, como así también interpretar su ubicación anatómica. En general, presentan diversa morfología, con tamaños y formas que varían desde grandes osteodermos subcuadrangulares de aproximadamente $40 \mathrm{~mm}$ por $30 \mathrm{~mm}$ hasta pequeńos subcirculares o elípticos de $8 \mathrm{~mm}$ por $7 \mathrm{~mm}$, ornamentados con pequeñas fosetas de distribución irregular (Fig. 1). Ninguno de los osteodermos recuperados supera los $4 \mathrm{~mm}$ de espesor. La región dorsosacra del ejemplar (Fig. 2) esta pobremente preservada y presenta dos filas de osteodermos paravertebrales, una de ellas integrada por siete osteodermos rectangulares. Además, se ha preservado la región proximal caudal con su escudo ventral articulado, el cual está integrado por cinco filas de osteodermos, exhibiendo la más completa de ellas aproximadamente once elementos (Fig. 4). Excepcionalmente, el ejemplar ha preservado asociado al fémur derecho una serie de pequeños osteodermos de forma subcircular a elíptica (Fig. 5).

Osteodermos dorsales. Se ha recuperado parte de la región dorsosacra de los osteodermos dorsales articulados (Figs. 2 y 3). Aunque el material se encuentra pobremente preservado, se pueden observar dos filas paravertebrales de osteodermos; la hilera paravertebral derecha representada por seis osteodermos mientras que la hilera izquierda compuesta por siete elementos (Fig. 2.2). Los osteodermos son rectangulares, con su lado mayor ubicado perpendicular al plano axial. La ornamentación de estos osteodermos está compuesta por fosetas de distribución irregular. En su borde anterior, los osteodermos poseen una faceta articular exterior no ornamentada de aproximadamente $4 \mathrm{~mm}$ de ancho (Figs. 1.1 y 2.2) —faceta para el ligamento interosteodermico- la cual se corresponde con la faceta articular inferior posterior del osteodermo precedente (sensu Salisbury y Frey, 2001). Esto indica que cada osteodermo anterior se hallaba imbricado o superpuesto sobre el osteodermo posterior. Otros elementos dorsales aislados presentan el mismo tipo de morfología y ornamentación, además de una baja cresta longitudinal que se proyecta en forma oblicua respecto del plano axial. Estos osteodermos de la región dorso-sacra son similares a los descritos por Fiorelli (2010) para el espécimen referido a Peirosauridae de la localidad del Campus Universitario, Formación Bajo de la Carpa, Ciudad de Neuquén. En algunos de ellos la cresta longitudinal se encuentra casi paralela al plano axial, ubicándose cerca de uno de sus bordes laterales. Los primeros 
cuatro osteodermos izquierdos de la región dorsosacra (Fig. 3.2), los cuales se hallan completos, poseen como el ejemplar MUCPv-27 (Fiorelli, 2010) una inflexión en su tercio lateral a nivel de la carena dermostealis (quilla), quedando divididos en dos sectores correspondientes a la parte medial y lateral (pars medialis y pars laterales sensu Salisbury y Frey, 2001). Uno de los osteodermos que fueron hallados aislados (Fig. 1.2-3), posee una notoria cresta o espina baja y de ápice redondeado, la cual, se encuentra inclinada posteriormente. En el notosuchio Simosuchus clarki Buckley, Brochu, Krause y Pol, 2000 sensu Hill, 2010, los osteodermos accesorios, al igual que los osteodermos paravertebrales poseen una cres- ta o espina, que adquiere un mayor desarrollo cuanto más lateralmente se encuentren ubicados en el escudo dérmico dorsal. Sin embargo, las crestas de los osteodermos hallados asociados a los miembros de Simosuchus clarki poseen un desarrollo aún mayor. En el caso del ejemplar MAU-Pv$\mathrm{PH}-437$, la morfología de los osteodermos apendiculares re-

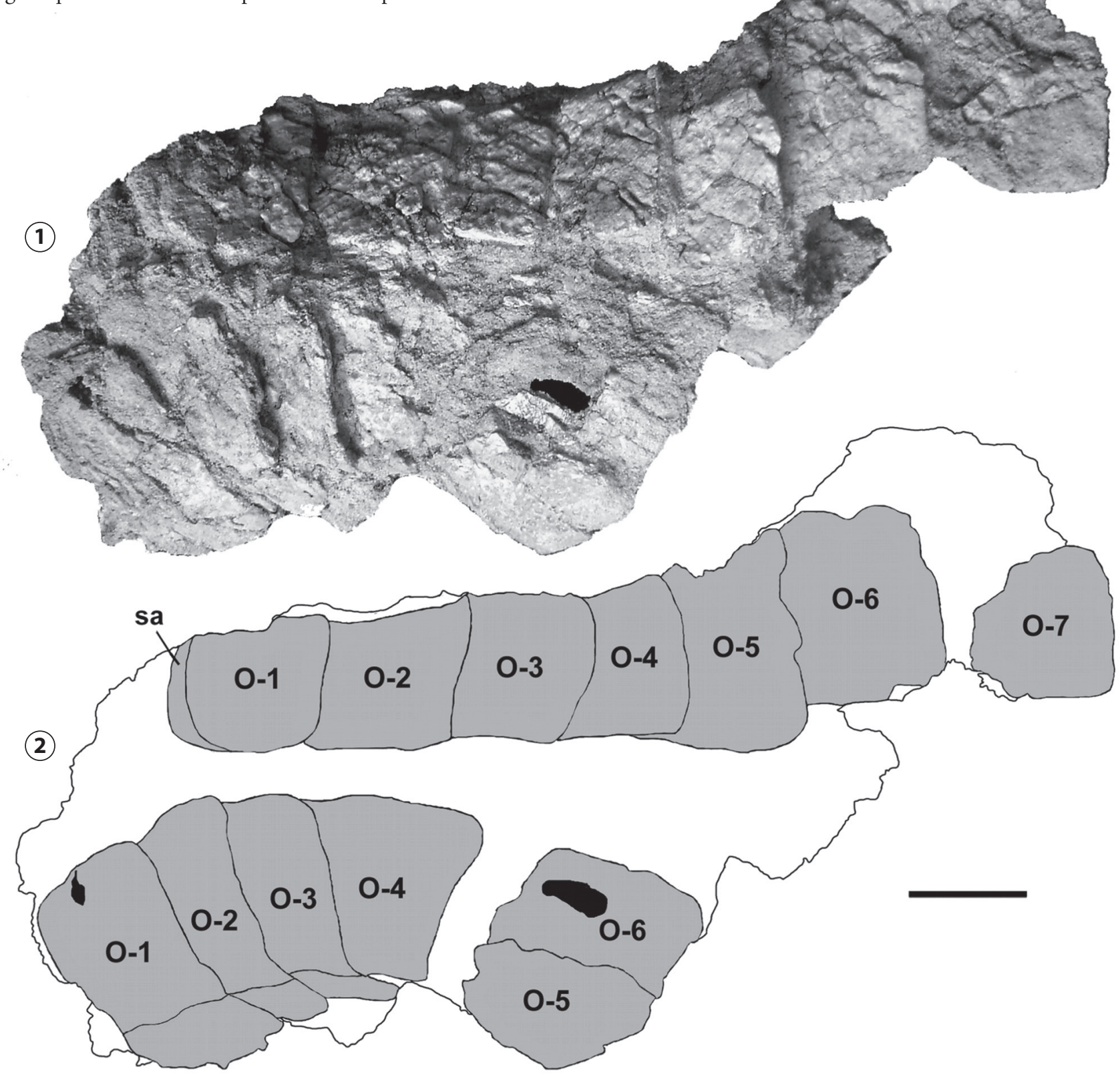

Figura 2. 1-2, vista dorsal de región dorso-sacra de osteodermos paravertebrales. Abreviaturas: 0-1 a 0-7, osteodermos 1 a 7; fa, faceta articular/ 1-2, Dorsal view of dorso-sacra region of paravertebral osteoderms. References: 0-1 to 0-7, osteoderms 1 to 7; fa, articular surface. Escala/ Scale= $2 \mathrm{~cm}$. 
sulta significativamente diferente a la presente en Simosuchus clarki. Por ello podemos inferir que dicho osteodermo no habría sido parte de los osteodermos apendiculares, sino que habría coronado la región dorsolateral de su tronco, formando hileras paralelas de osteodermos accesorios.
Osteodermos caudales. La sección proximoventral del escudo ventral caudal presenta dos hileras de osteodermos parasagitales una de ellas integrada aproximadamente por 11 osteodermos, y dos hileras laterales incompletas de osteodermos parasagitales accesorios (Fig. 4.1). En la región próxima a la

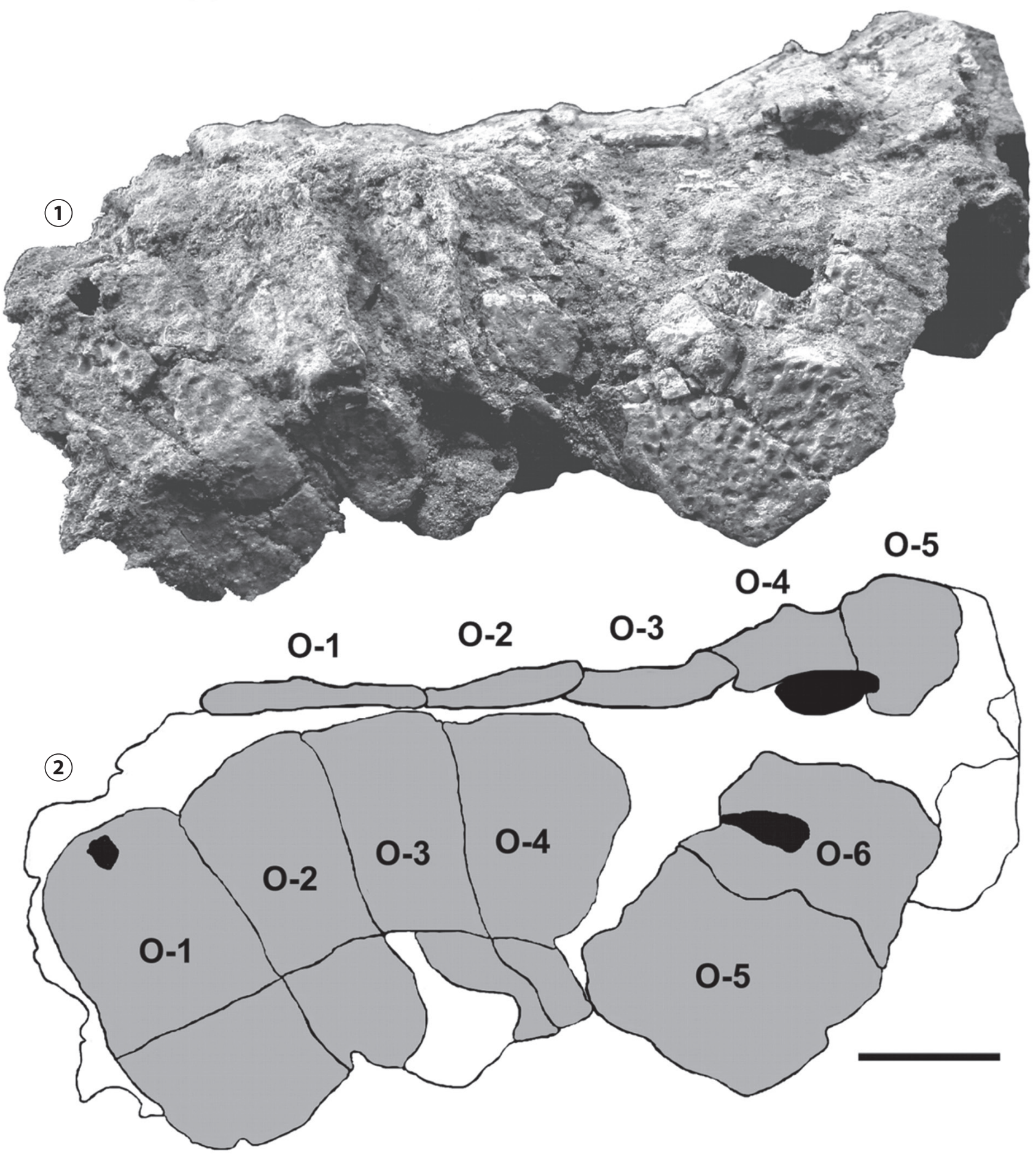

Figura 3. 1-2, Vista dorsal de los osteodermos paravertebrales del flanco izquierdo de la región dorso-sacra. Abreviaturas: 0-1 a 0-6, osteodermo 1 a 0-6/ 1-2, Dorsal view of paravertebral osteoderms to left side of dorsal sacral region. References: 0-1 to 0-6: osteoderms 1 to 6; fa, articular surface. Escala/ Scale $=2 \mathrm{~cm}$. 
pelvis se observa que el escudo ventral posee una serie de cuatro osteodermos de contornos subcirculares que pertenecerían por su tamaño $(7,8 \mathrm{~mm}$ a $21,2 \mathrm{~mm}$ de diámetro mayor) a la región cloacal o articular del miembro posterior. Los osteodermos parasagitales no poseen quilla. Sin embargo, los osteodermos parasagitales accesorios poseen una quilla subcentral orientada de forma oblicua respecto al plano axial (Fig. 4.2). Los osteodermos parasagitales caudales del escudo ventral son proximalmente subcuadrangulares $(22,3 \times 20,8 \mathrm{~mm})$, mientras que hacia el extremo distal de la sección caudal estos osteodermos disminuyen progresivamente su ancho, haciéndose rectangulares, con 17,6 mm de ancho por 29,3 mm de longitud. Los osteodermos parasagitales accesorios del escudo ventral son rectangulares, con sus bordes laterales redondeados. Como ocurre en los osteodermos dorsales del tronco, se observa en el borde anterior de los mismos una faceta articular no ornamentada para el osteodermo precedente.

Osteodermos apendiculares. Los osteodermos apendiculares fueron identificados y relacionados con su ubicación anatómica sobre la base de su morfología y su íntima asociación con uno de los huesos apendiculares. Asociado al fémur derecho (Fig. 5), se preservó una serie de aproximadamente 14 pequeños osteodermos de contorno subcircular a subelíptico, cuyos diámetros mayores varían entre 7,8 mm y 10,2 mm. Estos osteodermos se ubican sobre la mitad de la diáfisis del fémur, dejando ver solo sus caras internas, cuya superficie es lisa y prácticamente plana (Fig. 5.2). La cara externa, observada en otros osteodermos apendiculares hallados aislados, carece de cresta y presenta una ornamentación formada por fosetas subcirculares de distribución irregular (Fig. 1.5). Los osteodermos apendiculares
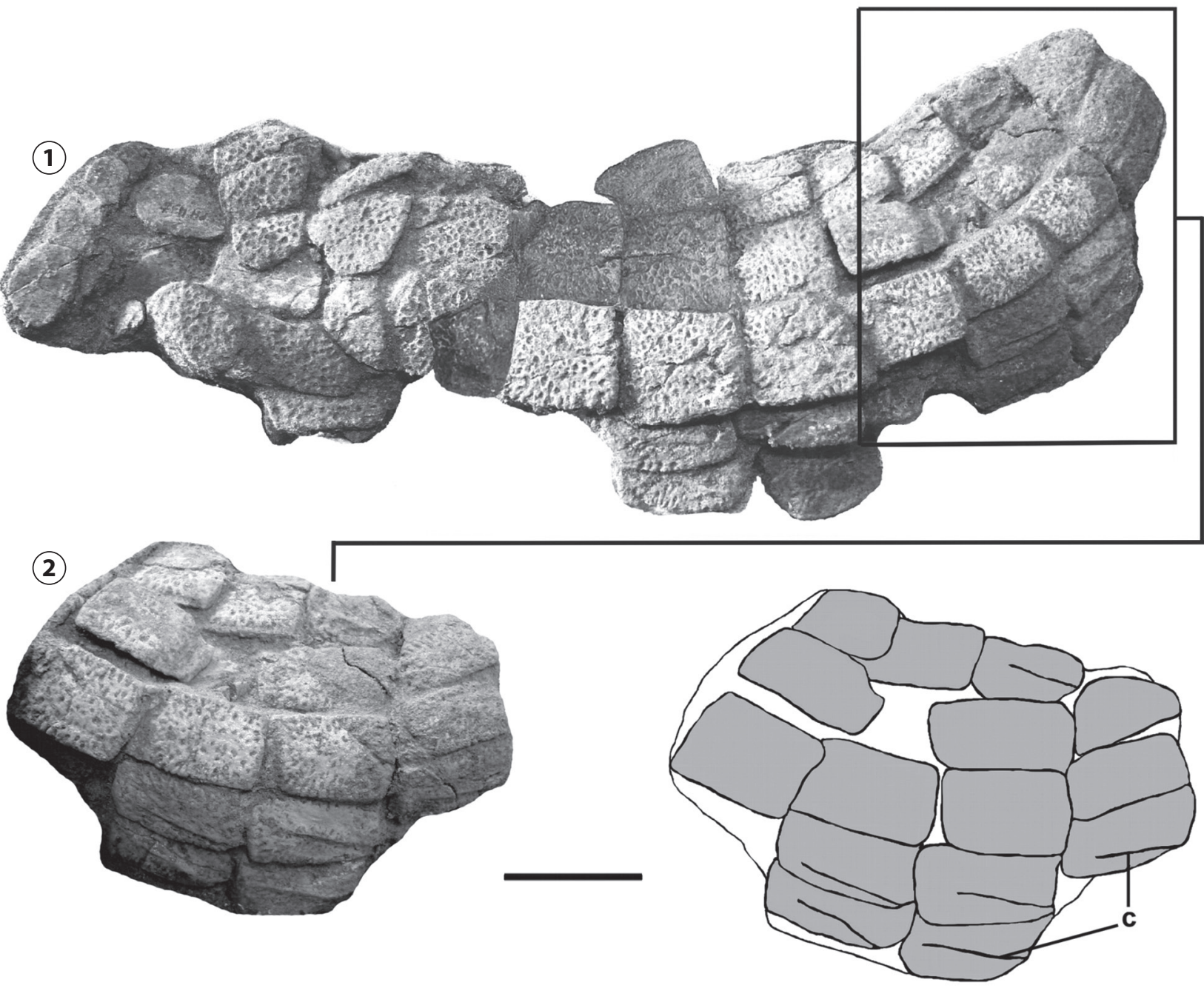

Figura 4. 1, vista ventral de la región proximocaudal mostrando el escudo de osteodermos ventrales; $\mathbf{2}$, detalle de los osteodermos caudales accesorios. Abreviaturas: c, cresta/ 1, ventral view of proximocaudal region showing the ventral osteoderm shield; 2, Detail of accesory caudal osteoderms. References: $c$, keel.Escala/ Scale $=2 \mathrm{~cm}$. 
asociados al fémur carecen de superficies de articulación entre sí, por lo que no se encontrarían imbricados sino asociados unos a otros para de esta manera cubrir los miembros del ejemplar. El tamaño de los osteodermos apendiculares varía en su diámetro mayor desde $5 \mathrm{~mm}$ a $15 \mathrm{~mm}$. Los osteodermos asociados al fémur indican que, en este ejemplar, los miembros estaban cubiertos por este tipo de protección dérmica.

Histología de los osteodermos. Dado que los estudios paleohistológicos en osteodermos de arcosaurios han demostrado ser de gran utilidad para la obtención de información paleobiológica, particularmente en lo que respecta a la determinación de la edad de un individuo (Erickson y Brochu, 1999; Hill y Lucas, 2006), el estudio morfológico de MAU-Pv-PH-437 ha sido complementado con un estudio histológico de sus osteodermos. Para tal fin se efectuaron secciones delgadas de cuatro osteodermos aislados del tronco (dos accesorios y dos dorsales paravertebrales), las cuales fueron analizadas mediante lupa binocular y microscopio petrográfico, utilizando tanto luz normal transmitida como luz polarizada. La nomenclatura histológica empleada corresponde a Chinsamy-Turan (2005).

La histología de los osteodermos de MAU-Pv-PH-437 se asemeja a la descripta previamente para otros crocodilomorfos (Hua y Buffrénil, 1996; Scheyer y Sander, 2004; Hill y Lucas, 2006; Vickaryous y Hall, 2008; Klein et al., 2009). Los osteodermos están constituidos por una corteza compacta que circunda una región interna más porosa (Fig. 6.1-2). En la corteza basal predomina el tejido pseudolamelar (parallel-fibred bone tissue), el

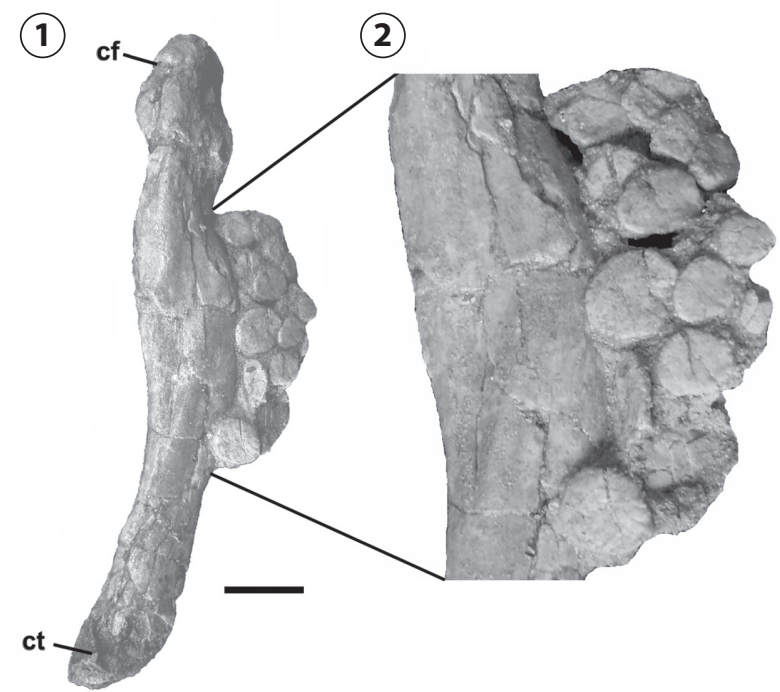

Figura 5. 1, Vista ventral de los osteodermos apendiculares asociados al fémur derecho; $\mathbf{2}$, detalle de los osteodermos apendiculares. Abreviaturas: cf; cabeza femoral; ct, cóndilo tibial/ 1, Ventral view of associated femur apendicular osteoderms; 2, detail of apendicular osteoderms. References: cf, femoral head; ct, tibial condyle. Escala/Scale $=2 \mathrm{~cm}$. cual se caracteriza por ser avascular y fuertemente birrefringente bajo luz polarizada (Fig. 6.3). Las lagunas de células óseas tienen aspecto alargado y su orientación es paralela a la de las fibras que las contienen. En algunas secciones se distinguen espacios vasculares conectados a la superficie basal mediante un conspicuo forámen. En la región interna, el tejido pseudolamelar se encuentra interrumpido por espacios de reabsorción de distinto tamaño, algunos de los cuales alcanzan una amplitud de $1 \mathrm{~mm}$. Los espacios de reabsorción pueden ser simples o estar revestidos por tejido lamelar. La corteza externa es avascular y se caracteriza por la presencia diversas capas de tejido lamelar correspondientes a distintos ciclos de reabsorción y nueva depositación ósea (Fig. 6.4) (Buffrénil, 1982). Este rasgo se vuelve más notorio en aquellos osteodermos cuya superficie externa se encuentra más ornamentada. Las distintas generaciones de tejido lamelar están claramente delimitadas por líneas de reabsorción. Como rasgo destacable, uno de los osteodermos estudiados reveló la presencia de tejido fibrolamelar en la corteza externa, incluso por encima de del tejido lamelar y separado de éste por una línea

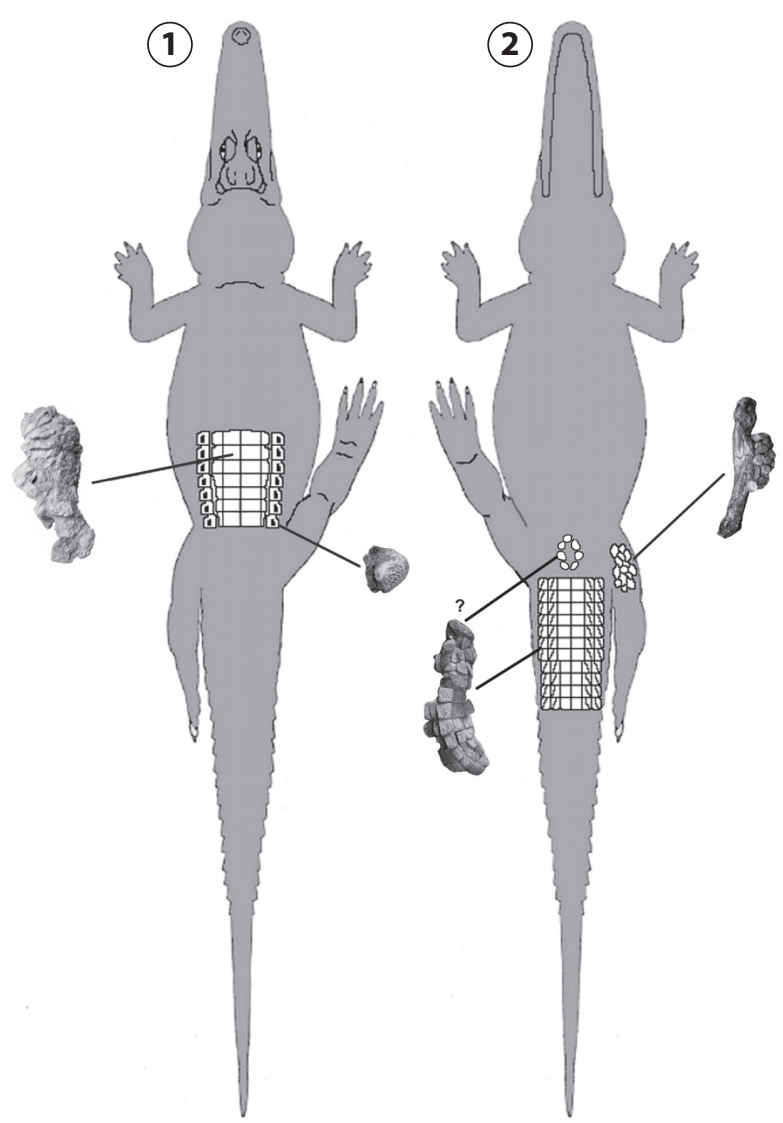

Figura 6. Reconstrucción esquemática de los osteodermos. 1, vista dorsal; 2 vista ventral (modificado de Salisbury et al., 2003)/ Schematic reconstruction of osteoderms. 1, Dorsal view; 2 , ventral view (modified from Salisbury et al., 2003). 
de reabsorción. Si bien este tipo de tejido ha sido previamente documentado en osteodermos de otros crocodilimorfos (Hill y Lucas 2006; Vickaryous y Hall 2008), su posición está comúnmente relegada a la región interna del elemento, no a la corteza externa y menos aún por encima del tejido lamelar.

Las fibras de Sharpey son comunes tanto en la corteza basal como en la externa, aunque su densidad, forma y disposición varía en las distintas regiones. En la corteza basal, las fibras de Sharpey aparecen como hebras delgadas y largas, en ocasiones agrupadas de forma laxa, distribuidas de manera espaciada (Fig. 6.5). Hacia la región media del osteodermo las fibras se orientan formado ángulos más bien rectos con la superficie externa. Hacia la región marginal de los osteodermos, dichos ángulos se vuelven más agudos. En la región de transición entre la corteza basal y externa las fibras extrínsecas se agrupan de forma más densa obscureciendo el patrón birrefringente del tejido pseudolamelar. De forma similar, las fibras extrínsecas de la corteza externa se distribuyen de forma más densa que en la corteza basal, siendo a la vez notoriamente más cortas (Fig. 6.4).

Es posible reconocer marcas de crecimiento (annuli en este caso) en prácticamente todo el tejido compacto, siendo en la corteza basal donde dichas marcas son más distinguibles. En este sentido, el continuo proceso de reabsorción y depositación ósea sufrido por la corteza externa, así como la presencia de falsas marcas de crecimiento (líneas de reabsorción, líneas entre lamelas sucesivas), impiden un conteo confiable de estas márcas en la corteza externa. En la serie mejor preservada de marcas de crecimiento, observada en un osteodermo accesorio (Fig. 6.6), se pudo contar un total de 17 annuli.

\section{DISCUSIÓN Y CONCLUSIONES}

La evolución de la armadura dérmica en crocodyliformes ha sido compleja y variada. Los arcosaurios basales como $\mathrm{Er}$ petosuchus granti Benton y Walker, 2002, así como también los crocodilomorfos basales Dromicosuchus grallator Sues, Olsen, Carter y Scott, 2003, Hesperosuchus agilis Clark, Sues y Berman, 2000, Junggarsuchus sloani Clark Xu, Forster y Wang, 2004, y Dibothrosuchus elaprhos Wu y Chatterjee, 1993, presentan una armadura dérmica formada por dos filas de osteodermos dorsales paravertebrales, cuya superficie dorsal se encuentra esculpida por fosetas irregulares separadas por crestas radiales originadas de una cresta longitudinal. Protosuchus richardsoni Colbert y Mook, 1951, presenta una armadura dérmica dorsal similar, la cual se extiende desde la nuca hasta el extremo distal de la cola, mientras que ésta última se encuentra rodeada completamente por osteodermos. Algunas formas intermedias de Mesoeucrocodylia, como los notosuquios Araripesuchus patagonicus Ortega,
Gasparini, Buscalioni y Calvo, 2000; Anatosuchus minor Sereno y Larsson, 2009; Notosuchus terrestris Woodward, 1896, y Gasparini, 1971 o Mariliasuchus amarali Carvalho y Bertini, 1999, poseen dos hileras paravertebrales de osteodermos. En Araripesuchus wegeneri Buffetaut y Taquet, 1979, al igual que el crocodiliforme basal Protosuchus richardsoni, Colbert, 1951, la región dorsal del tronco está cubierta por dos hileras de osteodermos paravertebrales y la región caudal está completamente rodeada de osteodermos.

En algunos Neosuchia basales, como los Atoposauridae, Pholidosauridae y Goniopholidae, la mayoría posee tan solo dos líneas de osteodermos dorsales en todo el cuerpo. En cambio en formas de Neosuchia más derivadas como Susisuchus anatoceps Salisbury, Frey, Martill y Buchy, 2003 e Isisfordia duncani Salisbury Molnar, Frey y Willis, 2006, además del registro de cuatro pares de osteodermos nucales, poseen una tetraserie paravertebral de osteodermos y, sobre ambos lados, una fila simple de osteodermos accesorios. La tetraserie de osteodermos que se continúa en la región caudal en Isisfordia duncani posee, además, dos filas longitudinales de osteodermos caudales accesorios que generan una doble cresta (Salisbury et al., 2006). En los Eusuchia como Akanthosuchus langstoni O’Neill, Lucas, y Kues, 1981, del Paleoceno de Nueva Mexico, se observa una gran variedad de osteodermos, los que fueron descriptos en detalle en un reciente trabajo de Hill y Lucas (2006), desde morfotipos cuadrangulares y ovales, hasta morfotipos con crestas y espinas bien desarrolladas.

La distribución de los osteodermos apendiculares entre los crocodyliformes no ha sido estudiada detalladamente ( $\mathrm{Pol}$ y Norell, 2004); por ello su importancia filogenética es aún incierta (Pol et al., 2009). Sin embargo, existen registros en algunos taxa dispares como el Goniopholididae Sunosuchus junggarensis Wu, Brinkman y Russell, 1996, los alligatóridos (Cong et al., 1998), los crocodyliformes basales Gobiosuchus kielanae Osmólska, Hua y Buffetaut, 1997, y Zaraasuchus shepardi Pol y Norell, 2004, y el notosúquido Simosuchus clarki. En los Gobiosuchidae, tales como Gobiosuchus kielanae, los osteodermos apendiculares están integrados por un mosaico de osteodermos cuadrangulares unidos mediante suturas y ornamentados por una delicada espina rodeada por cortos surcos radiales. Por otro lado, Zaarasuchus shepardi (otro Gobiosuchidae) posee osteodermos apendiculares pequeños $y$ de forma subcuadrangular sobre la región proximal del miembro anterior; estos osteodermos apendiculares están provistos de surcos poco profundos y una somera cresta. Además, posee osteodermos menos ornamentados y elongados en la región distal del miembro anterior. En el Goniopholididae Sunosuchus junggarensis, los osteodermos 

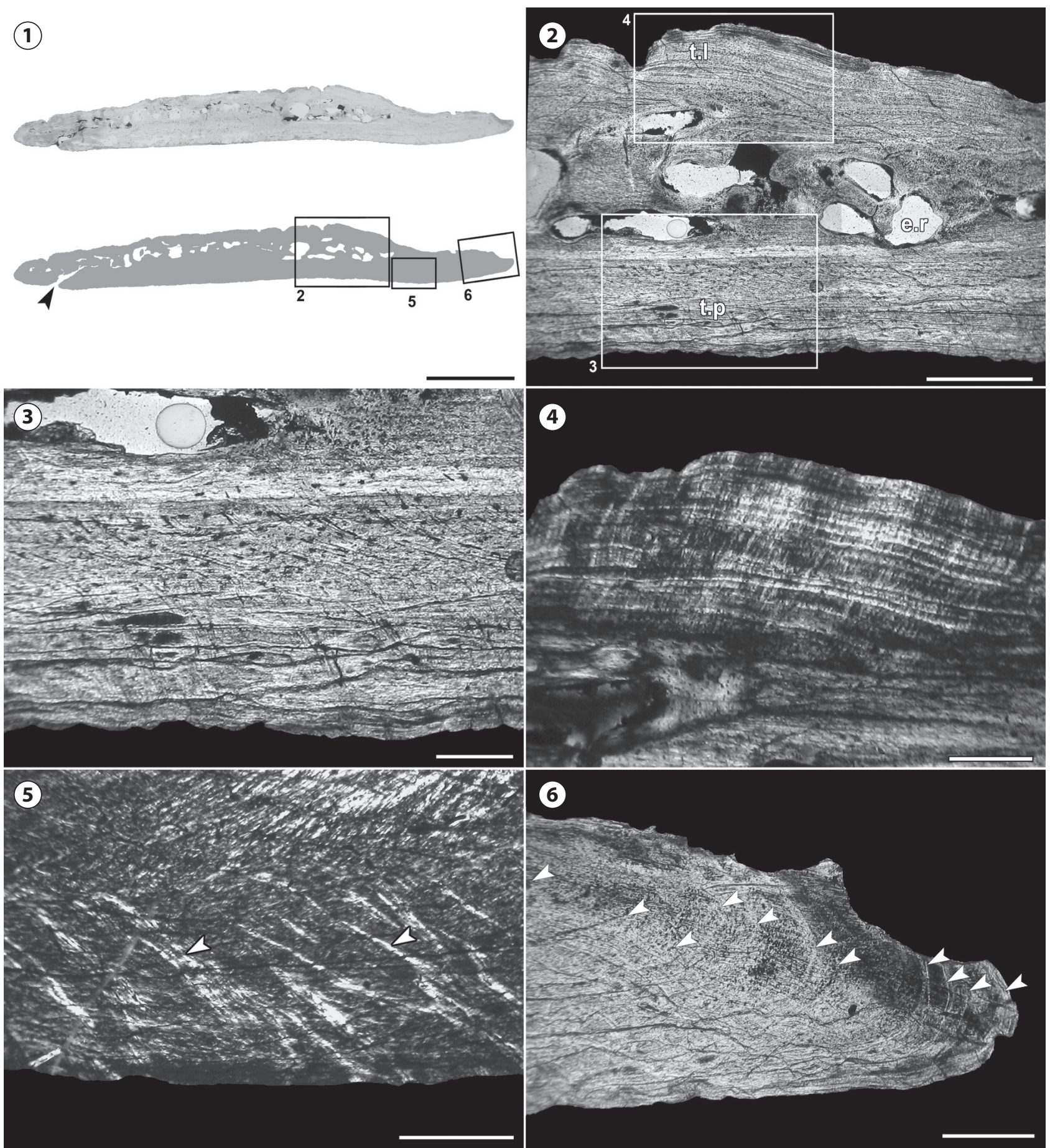

Figura 7. Microanatomía e histología de osteodermos. 1, aspecto general de la sección longitudinal de un osteodermo accesorio (arriba) y esquema del mismo corte donde se muestra la distribución del tejido compacto y espacios de reabsorción (abajo). Nótese la presencia de un foramen vascular en la corteza basal (flecha); $\mathbf{2}$, Detalle de la sección delgada donde se aprecia tejido pseudolamelar (t.p.) de la corteza basal, tejido lamelar (t.l.) de la corteza externa, y espacios de reabsorción internos (e.r.); $\mathbf{3}$, detalle del tejido pseudolamelar en la corteza basal; 4, tejido lamelar de la corteza externa invadido por numerosas fibras de Sharpey orientadas de forma perpendicular a la superficie externa; 5, fibras de Sharpey en la corteza basal. 6, marcas de crecimiento (flechas) en el extremo anterior del osteodermo. 1, 2, 3 y 5: luz normal, 4 y 6: luz polarizada/ Microanatomy and histology of MAU-Pv-PH-437 osteoderms. 1, general view of an accesory ostederm (top) and sketch drawing of the same section showing the distribiton distributon of the compact bone and resorption cavities (bottom). Note the presence of a vascular foramen in the basal cortex (arrowhead); 2, detail of thin section showing the pseudollamellar bone tissue (t.p.) in the basal cortex, lamellar bone tissue (t.l.) in the external cortex and resorption cavities (e..r) in the internal core; $\mathbf{3}$, detail of the pseudolamellar bone tissue in the basal cortex 4, lamellar bone tissue in the external cortex penetrated by abundant Sharpey fibres oriented perpendicularly to the external surface; 5, Sharpey's fibres (arrowheads) in the basal cortex; 6, growth marks (arrowheads) in lateral margin of the osteoderm. Scale $=5 \mathrm{~mm}(1), 1 \mathrm{~mm}(2), 0,3 \mathrm{~mm}(3,4)$ and $0.5 \mathrm{~mm}(5,6) .1,2,3$ and 5: polarised light: 4 and 6: normal light. 
apendiculares son pequeños, ovales con forma de hoja, esculpidos con fosetas de distribución irregular. En los Notosuchia, el registro más completo corresponde a Simosuchus clarki, el que posee osteodermos apendiculares tanto en el miembro anterior como en el posterior, que van desde formas cuadrangulares a romboidales en la región proximal del miembro, a elípticas y lentiformes en la región distal. En el miembro anterior, sólo en la región proximal, los osteodermos están provistos de cresta y se hallan articulados mediante suturas, mientras que en la región distal se encuentran imbricados y las crestas están ausentes. En todo el miembro posterior, los osteodermos poseen crestas y se encuentran articulados mediante suturas.El ejemplar MAU-PvPH-437 presenta osteodermos apendiculares de contorno subcircular a subelíptico, dos filas paravertebrales de osteodermos dorsales que probablemente se encuentren asociadas a osteodermos dorsales accesorios del tronco, que presentan una notoria espina, y dos filas de osteodermos parasagitales del escudo ventral de la sección, caudal con dos filas de osteodermos accesorios que presentan una cresta oblicua. Todos los osteodermos preservados exhiben una superficie dorsal ornamentada por fosetas de distribución irregular, mientras que la superficie ventral es lisa con algunos forámenes vasculares.

Aunque los osteodermos caudales del ejemplar MAU-PvPH-437 son ventrales, éstos exhiben una notoria similitud con el ejemplar MUCPv-27 (Bonaparte, 1991; Fiorelli, 2010) de la Formación Bajo de la Carpa, integrado por restos craneanos y postcraneanos (incluyendo numerosos osteodermos presacros y la región caudal media-posterior integrada por 25 filas transversales de osteodermos). Sobre la base de un análisis filogenético, Fiorelli et al. (2007) y Fiorelli (2010) relacionaron estrechamente al ejemplar MUCPv-27 con los Peirosauridae, siendo más afín a formas tales como Lomasuchus palpebrosus Gasparini, Chiappe y Fernández, 1991, y Peirosaurus tormini Price, 1955.

En el espécimen aquí descripto y en el espécimen MUCPv-27, los osteodermos caudales, exhiben una importante reducción distal progresiva de tamaño. Proximalmente son cuadrangulares, mientras que distalmente se hacen rectangulares. Los osteodermos apendiculares del ejemplar MAU-Pv-PH-437 asociados al fémur son similares a los hallados en el ejemplar MUCPv-27, aunque en este último ejemplar estarían asociados a las costillas dorsales, encontrándose localizados en los laterales del tronco (Fiorelli, 2010).

$\mathrm{Al}$ igual que en otros Mesoeucrocodylia como Araripesuchus patagonicus Ortega, Gasparini, Buscalioni y Calvo, 2000; Uberabasuchus terrificus Carvalho, Ribeiro y Avila, 2004; Peirosaurus torminni Price, 1955; Mahajangasuchus insignis Buckley y Brochu, 1999, y Anatosuchus minor Sereno y Larsson, 2009, el ejemplar en estudio presenta osteodermos dorsales del tronco más anchos que largos con una faceta articular anterior no esculpida. Estos osteodermos se encuentran divididos por una cresta en dos partes (pars medialis y laterales), su parte lateral se flexiona ventralmente y se encuentra ligeramente expuesta lateralmente. En Bernissartia fagesi Dollo, 1883, dicha cresta divide en forma similar el osteodermo, pero no posee un inflexión que genere una angularidad. Mientras que los osteodermos caudales proximales poseen bordes cuadrangulares, los osteodermos caudales distales poseen bordes más redondeados. Al igual que en los araripesúquidos (Hecht, 1991; Ortega et al., 2000; Turner, 2006), los osteodermos paravertebrales del tronco de MAU-Pv$\mathrm{PH}-437$ no poseen un proceso articular anterior desarrollado, el cual sí fue descripto en crocodilomorfos basales (Crush, 1984; Wu y Chatterjee, 1993; Clark et al., 2000), protosúquidos (Colbert y Mook, 1951), Goniopholis simus Salisbury, Willis, Peitz y Sander, 1999, y pholidosaurios (Sereno et al., 2001). Este proceso fue probablemente adquirido y perdido por diversos grupos durante la evolución de los Crocodyliformes (Sereno y Larsson, 2009) por lo que representaría un carácter bastante homoplásico. El ejemplar MAU-Pv-PH-437 se diferencia de Notosuchus terrestris Woodward, 1896, ya que este último presenta dos filas paravertebrales de osteodermos pentagonales, los que poseen una cresta longitudinal centrada lateromedialmente y ornamentación basada en suaves crestas y surcos (Pol, 2005).

Si bien los estudios anatómicos y filogenéticos en Crocodyliformes se encuentran basados en elementos craneanos, en los últimos años se han incluido caracteres postcraneanos en algunas propuestas filogenéticas (Arcucci et al., 2011). El ejemplar MAU-Pv-PH-437 es asignado a Peirosauridae indet. debido a una combinación única de caracteres (apomórficos y plesiomórficos) que caracteriza a esta familia, los cuales son detallados a continuación: superficie de los osteodermos dorsales con fosetas, presente en Peirosaurimorpha (Carlvalho et al., 2004), y también en casi todos los neosuquios y en algunos crocodyliformes basales (Pol, com. pers.); osteodermos sin proyección anterior, presente en Peirosauroidea (Carlvalho et al., 2004), y compartido además por los Notosuchia (Pol, com. pers.); osteodermos dorsales del tronco tres veces más anchos que largos (Sereno et al., 2003; 61); y cuatro filas de osteodermos paravertebrales (dos filas de osteodermos accesorios) (Clark, 1994; Sereno et al., 2003; 63), presente en Peirosaurus tormini. Por otro lado, con el espécimen MUCPv-27, considerado por Fiorelli et al. (2007) y Fiorelli (2010) como un Peirosauridae, comparte una cola completamente rodeada de osteodermos y osteodermos caudales, con una importante reducción progresiva distal de tamaño. 
En cuanto a la microestructura de los osteodermos, los datos aportados en este trabajo permiten incrementar el conocimiento en cuanto a su histología en arcosaurios suquianos (particularmente en crocodilomorfos Mesoeucrocodylia). Tal como se mencionó previamente, la microanatomía e histología de los osteodermos de MAU-Pv-PH-437 se asemejan a las descriptas para otros crocodilomorfos (Hua y Buffrénil, 1996; Scheyer y Sander, 2004; Hill y Lucas, 2006; Vickaryous y Hall, 2008; Klein et al., 2009), revelando un patrón general más bien conservativo en dicho linaje. El mantenimiento en los caracteres microestructurales de estas estructuras dérmicas en Crocodylomorpha se encontraría ligado tanto por un origen común como por la función común que desempeñan.

Los datos histológicos aquí obtenidos también pueden ser utilizados para inferir la edad del ejemplar MAU-Pv-PH-437. El conteo de marcas de crecimiento en osteodermos de cocodrilos actuales ha demostrado ser de considerable importancia para determinar la edad de los individuos (Hutton, 1986; Tucker, 1997). Ha sido empleado como herramienta esqueletocronológica también para taxones extintos (Erickson y Brochu, 1999; Hill y Lucas, 2006). Para una determinación más correcta de la edad de un individuo a partir del conteo de marcas de crecimiento en osteodermos, debe tenerse en cuenta de que en estas estructuras aparecen verdaderamente osificadas después del primer ańo de crecimiento (Chiappe et al., 1998, Vickaryous y Hall 2008). Puesto que el mayor número de marcas de crecimiento registrado en este trabajo corresponde a 17 , es posible determinar una edad mínima de 18 años para el ejemplar MAU-Pv-PH-437. Comparando con datos de longevidad en crocodilomorfos actuales, la edad mínima inferida para MAUPv-PH-437 indica que se trata de un individuo adulto.

El estudio detallado de los osteodermos en este ejemplar representa un importante aporte que permitirá identificar y ubicar anatómicamente, en base a la morfología e histología, osteodermos que hayan sido hallados aislados. Aunque en el presente trabajo no se persiguen objetivos filogenéticos, los caracteres presentes en los osteodermos permiten asignar preliminarmente el ejemplar MAU-Pv-PH-437 a Mesoeucrocodylia, siendo posiblemente un Peirosauridae. Un estudio que tome en cuenta estos osteodermos junto con los restantes elementos del esqueleto postcraneal recuperados, permitirán confirmar o modificar esta asignación.

\section{AGRADECIMIENTOS}

Nuestro agradecimiento está dirigido a los técnicos A. Valenzuela (†2005) y S. Palomo, quienes hallaron el ejemplar en el año 2004, durante tareas de relevamiento paleontológico para la empresa Petrobras; a los técnicos A. Paillán y W. Barros por su participación en las tareas de rescate del material; a D. Pol y L. Fiorelli, y a un revisor anónimo, por la revisión crítica del manuscrito, la cual permitió enriquecerlo sustancialmente; y a la Municipalidad de Rincón de los Sauces, por el apoyo logístico brindado en el desarrollo de las actividades.

\section{BIBLIOGRAFÍA}

Arcucci, A.B., Filippi, L.S. y Calvo, J.O. 2011. Un nuevo Mesoeucrocodylia cretácico del norte de la Cuenca Neuquina, Argentina. Revista Brasilera de Paleontología 14:1-10.

Benton, M. J. y Walker, A.D. 2002. Erpetosuchus, a crocodile-like basal archosaur from the Late Triassic of Elgin, Scotland. En: D.B. Norman y D.J. Gower (Eds.), Archosaurian anatomy and palaeontology. Essays in memory of Alick D. Walker. Zoological Journal of the Linnean Society 136: 25-47.

Bonaparte, J.F. 1991. Los vertebrados fósiles de la Formación Río Colorado, de la ciudad de Neuquén y cercanías, Cretácico Superior, Argentina. Revista del Museo Argentino de Ciencias Naturales "Bernardino Rivadavia" 4: 16-124.

Buckley, G.A. y Brochu, C.A. 1999. An enigmatic crocodile from the Upper Cretaceous of Madagascar. Special Papers in Palaeontology 60: 149-175.

Buckley, G.A., Brochu, C.A., Krause, D.W. y Pol, D. 2000. A pug-nosed crocodyliform from the Late Cretaceous of Madagascar. Nature 405: 941-944.

Buffetaut, E., y Taquet, P. 1979. An early Cretaceous terrestrial crocodilian and the opening of the South Atlantic. Nature 280: 486-487.

Buffrénil, V. de. 1982. Morphogenesis and bone ornamentation in extant and extinct crocodilians. Zoomorphology 99: 155-166.

Carvalho, I.S. y Bertini, R.J. 1999. Mariliasuchus: um novo Crocodylomorpha (Notosuchia) do Cretáceo da Bacia Bauru, Brasil. Geología Colombiana 24: 83-105.

Carvalho, I., Ribeiro, L.C. y Avila, L. 2004. Uberabasuchus terrificus sp. nov., a new crocodylomorpha from the Bauru Basin (Upper Cretaceous) Brazil. Gondwana Research 7: 975-1002.

Chiappe, L.M., Coria, R.A., Dingus, L., Jackson, F., Chinsamy, A. y Fox, M. 1998. Sauropod dinosaur embryos from the Late Cretaceous of Patagonia. Nature 396: 258-261.

Chinsamy-Turan, A. 2005. The microstructure of dinosaur bone: deciphering biology with fine-sacale techniques. Johns Hopkins University Press, Baltimore, $195 \mathrm{p}$.

Clark, J. 1994. Patterns of evolution in Mesozoic Crocodyliformes. En: N.C. Fraser y H. D. Sues (Eds.), In the shadows of the dinosaurs: Early Mesozoic tetrapods. Cambridge University Press, Cambridge, p. 84-97.

Clark, M. J., Sues, H. y Berman, D. S. 2000. A new specimen of Hesperosuchus agilis from the Upper Triassic of New Mexico and the interrelationships of basal crocodylomorph archosaurs. Journal of Vertebrate Paleontology 20: 683-704.

Clark, M. J., Xu X., Forster C.A. y Wang Y. 2004. A Middle Jurassic sphenosuchian from China and the origin of the crocodylian skull. Nature 430: 1021-1024.

Colbert, E. H. y Mook, C.C. 1951. The ancestral crocodilian Protosuchus. Bulletin of the American Musem of Natural History 94: 143-182.

Cong, L., Hong, L., Wu, X. y Hou, J.1998. The gross anatomy of Alligator sinensis Fauvel. Beijing: Science Press. [en Chino]

Crush, P.J. 1984. A late Upper Triassic sphenosuchid crocodilian from Wales. Palaeontology 27: 131-157.

Dollo, L. 1883. Première note sur les crocodiliens de Bernissart [First note on the crocodilians of Bernissart]. Bulletin du Musée Royal d'Histoire Naturelle de Belgique 2: 309-338.

Erickson, G.M. y Brochu, C.M. 1999. How the "terror crocodile" grew so big. Nature 398: 205-206.

Fiorelli, L. E. 2010. Predation bite-marks on a peirosaurid crocodyliform from the Upper Cretaceous of Neuquén Province, Argentina. Ameghiniana 47: 387-400.

Fiorelli, L.E. and Calvo, J.O. 2007. The first "Protosuchian" (Archosauria: Crocodyliformes) from the Cretaceous (Santonian) of Gondwana. Arquivos do Museu Nacional, Rio de Janeiro 65: 417-459. 
Fiorelli, L.E. y Calvo, J.O. 2008. New remains of Notosuchus terrestris Woodward (Crocodyliformes: Mesoeucrocodylia) from Late Cretaceous (Santonian) of Neuquén, Patagonia, Argentina. Arquivos do Museu Nacional, Rio de Janeiro 66: 83-124.

Fiorelli, L.E., Pol, D., Porfiri, J. D., Calvo, J.O. y Juárez Valieri, R.D. 2007. Peirosaurid affinities of crocodyliform from the Bajo de La Carpa Formation, Upper Cretaceous, Neuquén. Ameghiniana, Suplemento Resúmenes 44: 94R.

Frey, E. y Salisbury, S. W. 2007. Crocodilians of the Crato Formation: evidence for enigmatic species. En: D. M. Martill, G. Bechly, R. F. Loveridge (Eds.), The Crato Fossil Beds of Brazil. Window into an Ancient World. Cambridge University Press, Cambridge, p. 463-474.

Gasparini, Z. B. 1971. Los Notosuchia del Cretácico de América del Sur como un nuevo infraorden de Mesosuchia (Crocodilia). Ameghiniana 8: 83-103.

Gasparini, Z.B., Chiappe, L.M. y Fernández, M. 1991. A new Senonian peirosaurid (Crocodylomorpha) from Argentina and a synopsis of the South American Cretaceous crocodilians. Journal of Vertebrate Paleontology 11: 316-333.

Hecht, M. 1991. Araripesuchus Price, 1959. En: J.G. Maisey (Ed.), Santana fossils: an illustrated atlas. T.F.H. Publications, Neptune, p. 342-347.

Hill, R.V. 2010. Osteoderms of Simosuchus clarki (Crocodyliformes: Notosuchia) from the Late Cretaceous of Madagascar. Journal of Vertebrate Paleontology 30(6, Supplement): 154-176.

Hill, R.V. y Lucas, S.G. 2006. New data on the anatomy and relationships of the Paleocene crocodylian Akanthosuchus langstoni. Acta Palaeontologica Polonica 51: 455-464.

Hua, S. y de Buffrenil, V. 1996. Bone histology as a clue in the interpretation of functional adaptations in the Thalattosuchia (Reptilia, Crocodylia). Journal of Vertebrate Paleontology 16: 703-717.

Hutton, J. M. 1986. Age determination of living Nile crocodiles from the cortical stratification of bone. Copeia 2: 332-341.

Klein, N., Scheyer, T. y Tütken, T. 2009. Skeletochronology and isotopic analysis of a captive individual of Alligator mississippiensis Daudin, 1802. Fossil Record 12: 121-131.

Marinho, T., Ribeiro, L.C.B. y Carvalho, I. S. 2006. Morfologia de osteodermos de crocodilomorfosdo Sítio Paleontológico de Peirópolis (Bacia Bauru, Cretáceo Superior). Anuário do Instituto de Geociências, Universidade Federal do Rio de Janeiro 29: 44-53.

Legarreta, L. y Gulisano, C.A. 1989. Análisis estratigráfico secuencial de la Cuenca Neuquina (Triásico superior - Terciario inferior). En: G. Chebli y L. Spalletti (Eds.), Cuencas Sedimentarias Argentinas, Serie Correlación Geológica (INSUGEO) 6: 221-243.

O’Neill, F.M., Lucas, S.G., y Kues, B.S. 1981. Akanthosuchus langstoni, crocodilian from the Nacimiento Formation (Paleocene, Torrejonian) of New Mexico. Journal of Paleontology 55: 340-352.

Ortega, F., Gasparini, Z., Buscalioni, A.D. y Calvo, J.O. 2000. New species of Araripesuchus (Crocodylomorpha, Mesoeucrocodylia) from the Lower Cretaceous of Patagonia (Argentina). Journal of Vertebrate Paleontology 20: 57-76.

Osmólska, H., Hua, S. y Buffetaut, E. 1997. Gobiosuchus kielanae (Protosuchia) from the Late Cretaceous of Mongolia: anatomy and relationships. Acta Paleontologica Polonica 42: 257-289.

Pol, D. 1999. [El esqueleto postcraneano de Notosuchus terrestris (Archosauria: Crocodyliformes) del Cretácico Superior de la Cuenca Neuquina y su información filogenética. Tesis de Licenciatura, Facultad de Ciencias Exactas y Naturales, Universidad de Buenos Aires. 160 p. Inédita]

Pol, D. 2003. New remains of Sphagesaurus hunei (Crocodylomorpha, Mesoeucrocodylia) from the Late Cretaceous of Brazil. Journal of Vertebrate Paleontology 24: 817-831.

Pol, D. 2005. Postcranial remains of Notosuchus terrestris (Archosauria:

Crocodyliformes) from the upper Cretaceous of Patagonia, Argentina. Ameghiniana 42: 1-17.

Pol, D. y Norell, M.A. 2004. A new crocodyliform from Zos Canyon, Mongolia. American Museum Novitates 3445: 1-36.

Pol, D., Turner, A.H. y Norell, M.A. 2009. Morphology of the Late Cretaceous crocodylomorph Shamosuchus djadochtaensis and a discussion of neosuchian phylogeny as related to the origin of Eusuchia. Bulletin of the American Museum of Natural History 324: 1-103.

Pierce, S. E. y Benton, M. J. 2006. Pelagosaurus typus Bronn, 1841 (Mesoeucrocodylia: Thalattosuchia) from the Upper Lias (Toarcian, Lower Jurassic) of Somerset, England. Journal of Vertebrate Paleontology 26: 621-635.

Price, L.I. 1955. Novos crocodilídeos dos arenitos da Série Bauru. Cretáceo do Estado de Minas Gerais. Anais da Academia Brasileira de Ciências 27: 487-498.

Salisbury, S., and E. Frey. 2001. A biomechanical transformation model for the evolution of semi-spheroidal articulations between adjoining vertebral bodies in crocodilians. En: G.C. Grigg, F. Seebacher y C.E. Franklin (Eds.), Crocodilian Biology and Evolution. Surrey Beatty and Sons, Chipping Norton, p. 85-134

Salisbury, S.W., Willis, P. M. A., Peitz, S. y Sander, P. M. 1999. The crocodilian Goniopholis simus from the Lower Cretaceous of north-western Germany. Special Papers in Palaeontology 60: 121-148.

Salisbury, S.W., Frey, E., Martill, D. M. y Buchy, M. 2003. A new crocodilian from the Lower Cretaceous Crato Formation of north-eastern Brazil. Palaeontographica Abteilung A 270: 3-47.

Salisbury, S.W., Molnar, R.E., Frey, E. y Willis, P. 2006. The origin of modern crocodyliforms: new evidence from the Cretaceous of Australia. Proceeding of the Royal Society of London B 273: 2439-2448.

Scheyer, T. M. y Sander, M. 2004. Histology of ankylosaur osteoderms: implications for systematics and function. Journal of Vertebrate Paleontology 24: 874-896.

Sereno, P.C., Larsson, H.C.E., Sidor, C.A. y Gado, B. 2001. The giant crocodyliform Sarcosuchus from the Cretaceous of Africa. Science 294: 1516-1519.

Sereno, P.C., Sidor, C.A. Larsson, H.C.E. Gado, B.. 2003. A new notosuchian from the Early Cretaceous of Niger. Journal of Vertebrate Paleontology 23:477-482.

Sereno, P.C. y Larsson, H.C. E. 2009. Cretaceous Crocodyliforms from the Sahara. ZooKeys 28: 1-143.

Sues, H., Olsen, P., Carter, J. G. y Scott, D.M. 2003. A new crocodylomorph archosaur from the Upper Triassic of North Carolina. Journal of Vertebrate Paleontology 23: 329-343.

Tucker, A. D. 1997. Validation of skeletochronology to determine age of freshwater crocodiles (Crocodylus johnstoni). Marine Freshwater Research 48: 343-351.

Turner, A.H. 2006. Osteology and phylogeny of a new species of Araripesuchus Crocodyliformes: Mesoeucrocodylia) from the Late Cretaceous of Madagascar. Historical Biology 18: 255-369.

Vickaryous, M.K. y Hall, B.K. 2008. Development of dermal skeleton in Alligator mississippiensis (Archosauria, Crocodylia) with comments on the homology of osteoderms. Journal of Morphology 269: 398-422.

Woodward, A. S. 1896. On two Mesozoic crocodilians Notosuchus (genus novum) and Cynodontosuchus (genus novum), from the Red Sandstones of the Territory of Neuquén (Argentina Republic). Anales del Museo de La Plata, Paleontología 4: 1-20.

Wu, X-C. y Chatterjee, S. 1993. Dibothrosuchus elaphros, a crocodylomorph from the Lower Jurassic of China and the phylogeny of the Sphenosuchia. Journal of Vertebrate Paleontology 13: 58-89.

Wu, X-C, Brinkman, D.B. y Russell, A.P. 1996. Sunosuchus junggarensis sp.nov. (Archosauria: Crocodyliformes) from the Upper Jurassic of Xinjiang, People's Republic of China. Canadian Journal of Earth Sciences 33: 606-630.

doi: 10.5710/AMGH.8.6.2012.430

Recibido: 10 de noviembre de 2010

Aceptado: 8 de junio de 2012 